\title{
La capacidad de análisis en la formación de trabajadores sociales: diseño de una rúbrica de evaluación de la competencia
}

\author{
The ability of analysis in the training of social workers: \\ Designing a rubric for assessment of competencies
}

\author{
Maria Núria PRAT BAU \\ Universitat de Barcelona \\ nuriaprat@ub.edu \\ Belén PARRa RAmajo \\ Universitat de Barcelona \\ belenparra@ub.edu
Antonio LÓPEZ RODRÍGUEZ
Universitat de Barcelona
tonilopez@ub.edu

Recibido: $24 / 07 / 2014$

Revisado: $25 / 07 / 2014$

Aceptado: 24/11/2014

Disponible on line: 10/12/2014

\section{Resumen}

En este artículo se presenta un instrumento de evaluación (rúbrica) específico para la adquisición de la competencia de pensamiento analítico en la formación de Trabajo Social, aplicable a las materias de áreas diversas y cursos del Grado de Trabajo Social. Se describe el proceso metodológico y el enfoque colaborativo de su construcción, impulsada por el Grupo de Innovación Docente Trans@net: transdisciplinariedad y aprendizaje en Trabajo Social de la Universidad de Barcelona. Este proyecto ha permitido avanzar a los docentes en su conceptualización y reducir la disparidad de los criterios evaluativos utilizados inicialmente. Aunar una visibilidad común ante los estudiantes al compartir un mismo instrumento entre asignaturas coetáneas y cursos diferentes. Favorecer la transversalidad al aplicarse a materias de distintas áreas de conocimiento y definir unas condiciones provisionales de aplicabilidad como: la vinculación a actividades de aprendizaje que acompañen su adquisición, la aplicación en ejercicios centrados específicamente o de forma prominente en el desarrollo de esta competencia, la necesidad de trabajar previamente con los estudiantes la comprensión de la competencia, el instrumento y las relaciones existentes entre los ítems de la rúbrica y el contexto del contenido de la asignatura y del ejercicio propuesto.

Palabras clave: Trabajo Social, innovación educativa, evaluación, competencia analítica, rúbrica.

\begin{abstract}
In this article we present assessment tool (rubric) for the acquisition of specific competence on analytical thinking in social work training. This tool can be applied to the topics of various areas and courses of the Degree of Social Work. We describe the methodological process and collaborative approach to the construction of the instrument, which has been driven by the Innovative Teaching Group Trans @ net: transdisciplinarity and learning in social work from the University of Barcelona.

This project has enabled progress to teachers in their conceptualization and reduces the disparity of evaluation criteria used initially. Moreover, this has allowed offering a common visibility to students sharing the same instrument between different subjects and courses coeval. It has also encouraged the mainstreaming when applied to materials of different areas of knowledge and to define interim applicability conditions as: linking learning activities accompanying the acquisition, application exercises focused specifically or prominently in the development of this competition. It's necessary to work previously with students understanding of instrument (the relations among rubric items) and the context of each subject and proposed exercise.
\end{abstract}

Keywords: Social Work, pedagogical innovation, assessment, analytic competence, rubric. 
Referencia normalizada: Prat Bau, M. N., Parra Ramajo, B. y López Rodríguez, A (2014): «La capacidad de análisis en la formación de trabajadores sociales: diseño de una rúbrica de evaluación de la competencia». Cuadernos de Trabajo Social, 27(2): 365-374.

Sumario: Introducción. 1. Aproximación a la competencia de pensamiento analítico. 2. La rúbrica, un instrumento formativo e evaluativo de la competencia. 3. Discusión. 4. Referencias bibliográficas.

\section{Introducción}

En el marco de la formación universitaria en Trabajo Social emergen debates acerca del reto de formar profesionales que no se acomoden a la gestión de los míseros recursos sociales, que los contextos políticos e institucionales (y también profesionales) los han convertido en mecanicistas e impersonales. Estos plantean la necesidad de que los profesionales del Trabajo Social puedan analizar con reflexividad la complejidad de situaciones, procesos y prácticas profesionales que reproducen y legitiman posiciones y relaciones de poder político, económico y de saber profesional generadoras de desigualdad, $\mathrm{y}$, a partir de ella, junto con la ciudadanía ir construyendo otras posibilidades que promuevan la justicia social (Pastor y Martínez, 2014).

A este debate se le añaden las conocidas controversias sobre la formación universitaria llena de saberes desarticulados, compartimentados, distantes y utilitaristas para el mercado laboral que, según Morin (2011), no permiten enfrentarse a los retos de la realidad actual multidimensional, transversal, polidisciplinaria, transnacional, global y planetaria (p. 48). Estos hitos interpelan a los docentes a ser más selectivos con los objetivos, contenidos y procesos de aprendizaje para formar estudiantes más competentes en reflexividad, capacidad de análisis y creatividad. De modo que construyan su identidad profesional basándose en un compromiso ético y político en esta nueva (o vieja) realidad. Sin duda, a nivel de contenidos, implica acercar más las aulas a la cotidianidad de la ciudadanía, a los movimientos sociales, y a las lógicas comunitarias de creación y autogestión de otras formas de economía; así como abordar en la práctica social creativa y en la investigación las inquietudes de los profesionales, recuperando las bases relacionales de la profesión que fundamentan su área de conocimiento, y le dotan de credibilidad especialmente en el contacto con personas en situación de exclusión.
Para los autores de este articulo, el trabajador y la trabajador social de hoy necesitan, sobre todo, saber pensar antes de actuar, tomar posiciones de forma crítica y fundamentada ante las realidades y problemas sociales, poniendo en juego el pensamiento analítico y reflexivo, así como activar las capacidades creativas para construir nuevos significados y, en consecuencia, co-construir acciones que den respuesta a las necesidades emergentes de un mundo complejo en que «nada se mueve y a la vez todo está en movimiento». A nuestro modo de entender, la naturaleza compleja de la realidad social justifica la necesidad de dominar las competencias instrumentales, con enfoques metodológicos dirigidos hacia este fin. Saber analizar significa desgranar lo complejo ( $\sin$ fragmentarlo) separando las múltiples variables convergentes, para pensar en sus relaciones, significados y acciones.

Precisamente el listado de competencias en las titulaciones universitarias es extenso; cuenta con no demasiadas herramientas para su medición (García San Pedro y Gairín, 2011); son escasas las publicaciones del ámbito educativo del Trabajo Social que aplican y desarrollan el pensamiento analítico como competencia genérica, instrumental y sobre la forma de enseñarla y operativizarla en el currículum formativo; contrasta con una extensa literatura acerca de las formas de pensamiento reflexivo como método formativo crítico y transformativo; en este momento es necesario compartir algunos de los elementos de reflexión y conceptualización producidos por la labor del grupo de innovación docente, cuyo objeto principal ha sido el trabajo de conceptualización, desarrollo y evaluación de la competencia de pensamiento analítico. En concreto, se presenta el desarrollo es una herramienta formativa y evaluativa que permite una mayor sistematización para conocer el logro de la competencia.

La puesta en marcha del Grado de Trabajo Social en la Universidad de Barcelona (2010) 
implicó una revisión de los planes docentes y nuestras prácticas desde una perspectiva más global y abierta en todo el currículum. En 2012 cristalizó la creación del grupo de innovación docente,Trans@net: transversalidadyaprendizaje en Trabajo Social, que dio sus primeros pasos con el abordaje de las competencias transversales (denominadas también genéricas) para posteriormente centrarse en la comprensión transdisciplinar de la competencia de pensamiento analítico, y su adquisición progresiva a lo largo del Grado con el Proyecto de Innovación Docente "Hilos conductores: transversalidad y pensamiento analítico en Trabajo Social» (2012-2014) ${ }^{1}$.

\section{Aproximación a la competencia de pensa- miento analítico}

Lo que propongo es muy sencillo: «nada más que pensar en lo que hacemos» (Arendt, 1993, p. 18). Una vez puesto en marcha el Grado de Trabajo Social, según las coordenadas propuestas por el Espacio Europeo de Educación Superior, la enseñanza por competencias ha supuesto un hito teórico y metodológico, un nuevo paradigma educativo no exento de controversias y debates en relación a la programación y evaluación de la educación superior basada en competencias. Este enfoque parte de la consideración de las profesiones como un conjunto de competencias, generales y especificas de cada una de ellas, que configuran su perfil especifico. Por ello, en un primer momento, es preciso concretar el sentido del concepto analítico y metodológico de la competencia.

Hager, Holland y Becket (2002) proponen una definición de competencias que diferencia dos dimensiones implícitas: la cualidad y la capacidad. Las competencias incluyen habilidades de pensamiento (razonamiento lógico y analítico, solución de problemas), habilidades de comunicación y de trabajo en equipo; así como también cualidades personales como la creatividad y la imaginación y, valores como la ética profesional y la tolerancia, entre otros.

Biemans, Nieuwenhuis, Poell, Mulder y Wesselink (2004) señalan que las competencias aúnan capacidades integradas, orientadas a la práctica, consistentes en conjuntos de estructu- ras de conocimiento, así como en habilidades cognitivas, interactivas, y actitudes y valores necesarios para llevar a cabo tareas y resolver problemas.

El proyecto Tuning, 2003 (Tuning Educational Structures in Europe) clasifica las competencias y las diferencia entre competencias genéricas o transversales, que son comunes a la mayoría de las titulaciones, y las competencias especificas de cada titulación que definen el perfil profesional especifico. La competencia de pensamiento analítico se engloba en el grupo de competencias genéricas o transversales de tipo instrumental, siguiendo la taxonomía propuesta por Poblete (2007). La competencia de pensamiento analítico se entiende como el comportamiento mental, basado en un enfoque metódico que nos permite descomponer situaciones complejas en sus partes constituyentes, identificar los elementos substantivos, contextualizarlos y agruparlos para interpretar la realidad (Llobet y Vilà, 2013).

Sin embargo la separación entre pensamiento analítico, pensamiento reflexivo y pensamiento crítico, al revisar la literatura sobre el tema, no acaba de formularse en las propuestas de algunos autores. En este sentido, señalar que en la Guía de evaluación de las competencias del área de las ciencias sociales se considera el pensamiento analítico y el pensamiento crítico como un mismo tipo de pensamiento (Gairín, 2009).

Esta cuestión nos conduce a proponer la difícil separación de los tres tipos de pensamiento en el nivel de su adquisición competencial. Cada profesión desarrolla un modo particular predominante de pensamiento, lo que significa que no usa un solo modo de pensamiento, al contrario, se requieren los demás tipos de pensamientos complementarios (Villa y Poblete, 2007, p. 59). Esto nos lleva a plantearnos una serie de interrogantes: ¿podemos identificar y describir la relación entre estos tres tipos de pensamiento en el Trabajo Social? ¿Es nuclear la adquisición de la competencia de pensamiento analítico en la profesión de Trabajo Social?

En esta línea un estudio reciente del Consejo General del Trabajo Social (2013) destaca que los trabajadores sociales en su ejercicio profesio-

${ }^{1}$ Proyecto de Innovación Docente 2011PID-UB/24: Fils conductors: transversalitat docent i pensament analític a Treball Social (GID, Trans@net), Facultad de Pedagogía. Universidad de Barcelona. 
nal identifican precisamente como ventajas de su formación el desarrollo de una gran capacidad de análisis (10 por ciento), ocupando el tercer lugar por debajo de otras más destacadas como la formación en diferentes ámbitos (sociología, psicología, economías, derecho, etc.) $(40,2$ por ciento) y las habilidades adquiridas para la resolución de problemas $(29,1$ por ciento). Estos resultados indican que la mirada profesional enfatiza los referentes y bases interpretativas teóricas y las habilidades resolutivas. Pero vemos la estrecha relación entre ellas, dado que las primeras son necesarias para interpretar los datos analíticos y la toma de decisiones rigurosa, requiere de herramientas metodológicas y procesuales previas de tipo analítico. Si aceptamos que los trabajadores sociales necesitan tomar importantes decisiones en contextos y circunstancias complejas, en relación a situaciones y relaciones sociales con diferentes dimensiones explicativas. Parece lógico que para poder pensar mejor esta realidad, utilicen procedimientos de naturaleza analítica como: descomponer los elementos constituyentes, identificar las variables que permitan obtener datos e interpretarlos, sabiendo que es una realidad representada e intersubjetiva, y tomar decisiones para la práctica.

Sin embargo, no existe acción compleja sin reflexión durante el proceso analítico; es necesaria la reflexión sobre la situación, los objetivos, lo medios necesarios, los resultados provisionales y la evolución previsible. La reflexión en la acción consiste en preguntarse: qué pasa o pasará, qué podemos hacer, qué hay que hacer, cuál es la mejor táctica, qué orientaciones y qué precauciones hay que tomar (Perrenoud 2004, pp. 30-31).

Por otra parte, no debemos olvidar que tanto el proceso analítico, como la práctica profesional que se deriva, se desarrollan en una relación intersubjetiva. En esta premisa se encuentra el nudo de conexión entre la competencia de pensamiento analítico y el pensamiento denominado reflexivo, porque la reflexión sobre la acción conlleva, como plantea Bárcena (2005, p. 54), la responsabilidad intelectual o la capacidad para responder de las consecuencias que lógicamente se deducen de una determinada proposición: ser intelectualmente responsable quiere decir considerar las consecuencias de un paso proyectado. Y, en la práctica profesional del Trabajo Social, «los pasos proyectados» se construyen en relaciones dialógicas.

Una práctica competente fruto de la interrelación entre el pensamiento analítico, el reflexivo y el crítico, implica en consecuencia, analizar las partes constituyentes de una realidad social dada, contextualizarlas y agruparlas, y simultáneamente analizar las aproximaciones al conocimiento que se utilizan y que orientaran las decisiones sobre la intervención y, de la misma manera interrogarse sobre la naturaleza de la transformación social de dichas intervenciones y sobre el carácter intersubjetivo de la relación profesional.

\section{La rúbrica, un instrumento formativo e evaluativo de la competencia}

Evaluar es una de las tareas más complejas que afronta el docente, sobre todo cuando en la educación superior tratamos de aprendizajes complejos que no pueden ser reducidos a un listado de criterios y estándares que el estudiante debe saber reproducir (o repetir).

Las rúbricas tienen una estructura matricial organizada de acuerdo con ámbitos o dimensiones de la competencia (columnas), junto con unos estándares de desempeño progresivos (filas), entendidos como el nivel de logro que se le requiere al estudiante para poder confirmar su adquisición de la competencia dentro del curriculum formativo (Hawes, 2004)². El estándar no solo establece los requisitos sino también admite los grados en los cuales puede fijarse la calidad de desempeño dentro de una escala cualificada que, en nuestro caso y contexto cultural educativo, se concreta en cuatro grados de logro (insuficiente, aprobado, notable y excelente) ${ }^{3}$.

\footnotetext{
${ }^{2}$ Este autor señala la importancia de que el desempeño de la competencia no se centre únicamente dentro del currículum formativo, sino que acredite también su logro en el ejercicio profesional. El instrumento presentado tiene limitaciones en este sentido. Precisamente una de las líneas de continuidad del proyecto pretendería validarlo en el ámbito profesional.

${ }^{3}$ En el diseño de la rúbrica aplicada en la prueba piloto se ha decidido temporalmente no mostrar la correspondencia numérica de la escala de cualificación a la espera de obtener unos primeros resultados sobre las puntuaciones obtenidas que orienten el debate realizado dentro del grupo de innovación.
} 


\begin{tabular}{|c|c|c|c|}
\hline $\begin{array}{c}1 \\
\text { Insuficiente }\end{array}$ & $\underset{2}{2}$ & $\begin{array}{c}3 \\
\text { Notariedad }\end{array}$ & $\begin{array}{c}4 \\
\text { Excelente }\end{array}$ \\
\hline $\begin{array}{l}\text { No cumple los requeri- } \\
\text { mientos del desempeño. } \\
\text { Logro insuficiente }\end{array}$ & $\begin{array}{l}\text { Acredita un nivel de des- } \\
\text { empeño suficiente en el } \\
\text { curriculum/ejercicio pro- } \\
\text { fesional }\end{array}$ & $\begin{array}{l}\text { Supera el nivel } \\
\text { de logro espera- } \\
\text { do }\end{array}$ & $\begin{array}{l}\text { Nivel excepcional de des- } \\
\text { empeño por encima de lo } \\
\text { esperado. Propone y de- } \\
\text { sarrolla nuevas acciones }\end{array}$ \\
\hline
\end{tabular}

Tabla 1. Escala general de apreciación del logro.

Fuente: Elaboración propia basada en Hawes (2004).

Cada nivel de logro se describe como una superación del anterior y establece una direccionalidad en términos de domino de menor (1) a mayor logro (4). A través de un laborioso trabajo de consenso se determina cuál es el desempeño aceptable y por debajo del cual no lo es, con toda su ambigüedad y prudencia, tal y como muestra la tabla 1.

\subsection{Materiales y métodos: descripción del proceso}

Participan en este proyecto un grupo de $10^{4}$ asignaturas. de las cuales 6 corresponden a primer curso y cuatro a segundo, con un total de 16 docentes participantes de procedencias disciplinares diversas con un mayor predominio del área de Trabajo Social (11).

En el grupo de innovación participan el profesor coordinador de cada asignatura y otro miembro del equipo docente, con la intención de garantizar siempre la presencia de un miembro al menos por asignatura. Los espacios de trabajo tienen una frecuencia mensual estable y se encuentran coordinados por dos profesoras. La mayor parte de las tareas del proceso de investigación se han realizado en subequipos y en espacios flexibles. El proceso de trabajo del grupo y del proyecto se documenta a través de actas, informes parciales y finales.

En relación a la descripción del proceso de trabajo se pueden distinguir cinco etapas consecutivas.

$1^{a}$ etapa (2010-2011): Conceptualización teórica de la competencia. Se realiza un proceso reflexivo a partir de la lectura de referentes teóricos, según apuntan Poblete (2007) y Moya (2008), que permiten el consenso de una definición.

$2^{\mathrm{a}}$ etapa (2011-2012): Análisis inductivo de la práctica docente. ¿Cómo evalúa el profesorado la capacidad de análisis de los estudiantes en sus trabajos académicos? Se realiza un trabajo exploratorio para conocer cómo evalúan los docentes la capacidad de análisis de los estudiantes en los ejercicios que proponen en sus asignaturas con esta finalidad. Se realiza una recogida sistemática de datos donde los profesores aportan un ejemplo de buena y mala práctica de pensamiento analítico, basándose en el ejercicio de un estudiante anónimo que ellos

\begin{tabular}{|c|l|}
\hline Etapas & \multicolumn{1}{c|}{ Descripción } \\
\hline $1^{\text {a }}$ Etapa (2010-2011) & Conceptualización teórica de la competencia \\
\hline $2^{\text {a }}$ Etapa (2011-2012) & Trabajos parciales de análisis inductivo de la práctica docente \\
\hline $3^{\text {a }}$ Etapa (2012-2013) & $\begin{array}{l}\text { Construcción de la rúbrica: descripción de los criterios y estándares de la com- } \\
\text { petencia y gradación de la adquisición }\end{array}$ \\
\hline $4^{\text {a }}$ Etapa (2013-2014) & Validación de la rúbrica en una prueba piloto \\
\hline
\end{tabular}

Tabla 2. Etapas de construcción de la rúbrica.

Fuente: Elaboración propia.

${ }^{4}$ A lo largo del proyecto ha habido variaciones en el número de asignaturas y profesores participantes debido a cambios organizativos dentro de los equipos docentes. En el 2011 participan 12 asignaturas y 16 profesores. En el 2013, participan 10 asignaturas 16 profesores. 
mismos han corregido, cuya valoración justifican. A través de este trabajo inductivo se extraen los criterios e ítems utilizados por el profesorado en la evaluación de la competencia.

$3^{\mathrm{a}}$ etapa (2012-13): Construcción de la rúbrica: descripción de los criterios y estándares de la competencia y su gradación para su adquisición. Se retoma el trabajo anterior y el marco teórico estudiado como base para iniciar la construcción de la rúbrica. Se operativizan los estándares dentro de una matriz graduada, con una escala de calificación cualitativa y cuantitativa (en proceso) de los resultados de desempeño de la competencia.

Se realiza una primera aplicación de la rúbrica sobre un ejercicio auténtico. Se diseña un ejercicio de análisis basándose en una situación social única construida por todo el equipo y se pide a los estudiantes de primer curso, segundo semestre que analicen la situación (texto) presentada, integrando los conocimientos de todas las asignaturas. El mismo ejercicio se aplica en dos tiempos distintos, febrero-mayo (al inicio y al final del semestre). Por limitaciones de tiempo, se escogen al azar 12 ejercicios de un total de 250 realizados. Se evalúan por parejas de profesores de disciplinas diferentes y se corrigen a ciegas, sin identificar los nombres de los autores ni el orden en que han sido realizados los ejercicios Los dos profesores correctores redactan un informe sobre la existencia de pensamiento analítico en el texto realizado por el estudiante y se justifica su valoración utilizando la primera versión de la rúbrica. Los resultados permiten revisar el instrumento, especialmente los ítems de adquisición, así como matizar algunas definiciones (Ibarz, Prat, Vives, 2013)

$4^{a}$ etapa (2013-14): Validación de la rúbrica en una prueba piloto. Esta se aplica en 8 asignaturas, 5 de las cuales corresponden al primer curso, 2 al segundo y 1 al tercero, formando un total de 14 grupos distintos ( 8 de mañana y 6 de tarde). En relación a las características de los ejercicios, 10 son en grupo y 4 individuales. El tipo de ejercicios donde se ha aplicado son diversos en objetivos competenciales. Encontramos, actividades globales extensas donde la capacidad de análisis confluye junto con otras competencias en equidad; otras, donde la capa- cidad para el análisis (CPA) confluye con otras competencias pero tienen un rol predominante $(\geq 80 \%)$; Y ejercicios más específicos dónde únicamente se considera la CPA (100 por ciento).

\subsection{Resultados: descripción del instrumen- to creado}

Como resultado principal se expone el instrumento creado (ver anexo 1) y se describen aportaciones de carácter conceptual, metodológicas sobre el proceso de elaboración del instrumento y se apuntan orientaciones para su aplicación en el Grado de Trabajo Social.

\section{Aportaciones conceptuales}

- Construcción de pensamiento múltiple y competencias interconectadas. Se constata el nudo de conexiones existente entre diversas formas de pensamiento (reflexivo, crítico, creativo, práctico, sistémico, etc.) y se ponen de relieve las dificultades y limitaciones de aislar y desgranar la competencia de análisis de las otras, dado que su tipología instrumental y carácter metodológico la convierten en una competencia procesual básica para tomar de decisiones fundamentadas.

\section{Aportaciones metodológicas}

- La utilización de práctica docente como fuente inductiva de datos crea un marco colaborativo óptimo para la innovación educativa. Esta decisión procesual ha comportado una mayor lentitud de trabajo, pero ha conseguido un mayor conocimiento mutuo y de las materias, una mayor conciencia de la disparidad de criterios utilizados cuando evaluamos y la internalización de la complejidad del tema por parte del profesorado participante. Se destacan cuatro momentos clave: 1- Hacer un puesta en común de las concepciones previas sobre el pensamiento analítico. 2- Compartir la descripción de las actividades y ejercicios propuestos en cada asignatura para desarrollar la competencia de análisis. 3- Realizar la descripción de los criterios de corrección utilizados sobre un ejercicio de cada asignatura por parte del profesorado. 4- La creación de un texto-relato analizable desde los contenidos de todas las asignaturas, realización del análisis por parte de los estudiantes y la evaluación a ciegas entre pares de profesores de disciplinas diversas. 
- El proceso de trabajo como una estrategia hacia la trandisciplinariedad. La utilización de la reflexividad docente en los dos primeros cursos del grado, donde se halla el mayor cruce de materias de áreas de conocimiento diversas son factores que han logrado un mayor conocimiento entre profesorado; contenidos, metodologías y ejercicios entre las asignaturas simultáneas por semestres y cursos, posibilitando la creación de una cultura de trabajo favorecedora de la transversalidad entre los equipos docentes participantes y, por extensión, el resto de equipos del Grado.

Aportaciones de la rúbrica: oportunidades y límites del instrumento

- Es un instrumento sobre todo formativo. La rúbrica no puede ser aplicada como mero instrumento evaluativo, debe ir acompañado de actividades de aprendizaje que favorezcan su adquisición. La capacidad analítica no se adquiere espontáneamente, sino que necesita ejercitarse y requiere de espacios deliberados para este fin a lo largo de los estudios universitarios. A nuestro modo de entender es tan importante el instrumento como los ejercicios diseñados para su desarrollo. En el marco del proyecto se han recopilado los utilizados para su adquisición y queda abierta la posibilidad, para futuros proyectos, de la construcción de un mapa competencial dentro de la titulación.

- Es necesario asegurar la comprensión de la competencia y del instrumento por parte de los estudiantes antes de la aplicación del instrumento. Los estudiantes necesitan entender el instrumento y los requerimientos del pensamiento analítico en cada grado de adquisición. Hay que dialogar con ellos sobre la descripción de los ítems y su contextualización en los contenidos de la asignatura y el ejercicio propuesto, con ejemplos comprensibles que orienten la ejecución de la tarea y la claridad de los niveles de dominio.

- Se requiere explicitar las competencias implicadas en las tareas de aprendizaje. Se valora que los docentes construyan las actividades de aprendizaje, precisando las competencias que se trabajarán en cada tarea en concreto, y el peso que tiene la CPA dentro de ellas. El instrumento se adapta mejor a tareas específicas donde la competencia analítica se trabaja exclusivamente, o tiene un peso igual o superior al 80 por ciento de la nota final.

- Incrementa la autonomía y autorregulación del aprendizaje. A partir de las fuentes teóricas se presupone que la rúbrica guía y orienta el trabajo que los estudiantes tienen que realizar y les permite mejorar la percepción sobre el logro durante el proceso de realización (Picornell-Lucas, 2014). También el profesorado la utiliza para justificar su cualificación y orientar los puntos de mejora.

- Es un instrumento válido tanto en actividades de carácter individual como grupal. En este último caso, hay que tener presente que la capacidad analítica evaluada corresponde al proceso de trabajo del grupo y se entrecruza la competencia de trabajo en equipo.

- Tiene un mejor funcionamiento en actividades de aprendizaje extensas, complejas y con objetivos específicos centrados en el desarrollo de la CPA. Un ejercicio extenso y global permite el desarrollo de todos los ámbitos incluidos en la rúbrica (descomposición, ordenación, categorización, interpretación, conclusión y apertura de nuevos interrogantes). Se encuentran limitaciones importantes cuando la rúbrica se aplica sobre ejercicios simples, con tiempo limitado (30') y sin disponibilidad de acceso a referencias (fuentes teóricas o datos empíricos) que permitan desarrollar la dimensión interpretativa de la situación social expuesta.

- Permite consensuar la progresión de adquisición de la competencia a lo largo del grado. Los niveles de adquisición de la competencia deben ser diferentes para un estudiante de primer curso para otro de cuarto. La discusión colectiva ha permitido la concreción de un hito fijando el «aprobado mínimo» para los cursos de primero a tercero. Ahora el profesorado es más consciente de su papel y contribución a la adquisición progresiva de la competencia materializada en el Trabajo de fin de Grado del último curso.

- Es un instrumento evaluativo aún incompleto por falta de puntuaciones cuantitativas. Por ahora facilita la evaluación formativa y está previsto establecer una propuesta cuantitativa, una vez se obtengan los resultados de la prueba piloto. Por las pruebas realizadas, se destaca un buen funcionamiento de los ámbitos que incluye la competencia (columnas), pero es necesario perfilar mejor los niveles de logro progresivo (filas). 


\section{Discusión}

Uno de los puntos de mayor controversia ha sido la discusión sobre el grado de adaptación de la rúbrica. Mientras parece lógico que cualquier instrumento de medida, de tipo generalista, como el que se presenta, requiere una adaptación y un ajuste mínimo a las actividades de aprendizaje de las diversas asignaturas; la coherencia pedagógica nos dice que son los objetivos de aprendizaje los que tienen que determinar el diseño de actividades y en consecuencia los instrumento evaluativos. En esta experiencia el orden ha sido al contrario, la creación del instrumento ha sido posterior y se ha aplicado a las actividades que las asignaturas ya se venían desarrollando.

Se señala que este punto crítico podría considerarse un error de consistencia metodológica que debe tenerse presente en la interpretación de los resultados. Probablemente el hecho de que se trate de un proyecto a largo plazo y el propio proceso de inmersión y profundización realizado por el profesorado han inducido a que una parte de éstos haya hecho modificaciones en el diseño de sus ejercicios para focalizar mejor al estudiante y el análisis de situaciones sociales, antes de la aplicación de la rúbrica. Mientras que otra parte ha aplicado, en sentido estricto, la rúbrica sobre los ejercicios iniciales sin ningún tipo de adaptación previa, permitiendo una observación más clara de los límites del instrumento.

Aunque, como hemos señalado, estamos en el proceso de tratar los datos de la prueba piloto, en los resultados hemos avanzado que la aplicación del instrumento presenta mayor complejidad evaluativa cuando coexisten dos o más competencias implicadas en el ejercicio. Este resultado operativo es poco alentador ya que como hemos recogido en el marco teórico no podemos perder de vista la lógica del pensamiento múltiple (Gardner, 2005) y el contexto de la complejidad de las situaciones sociales al que se enfrentan los profesionales, que requieren coexistencia de las diversas formas de pensamien- to. Mientras retamos a los docentes a diseñar ejercicios que integren formas de pensar y competencias diversas. Disponemos de un instrumento que se adapta mejor a ejercicios monocompetenciales ¿Qué hacemos? Señalamos la necesidad de apostar por una perspectiva amplia de la competencia analítica. El ámbito del Trabajo Social nos exige dominar esta capacidad complementariamente con otras formas de pensamiento que la preceden o coexisten. Los trabajadores sociales cuando analizan deben hacerlo desde una posición reconocida y fundamentada (pensamiento crítico) y es importante que el análisis provenga de la reflexividad de la práctica profesional y teórica, manejando habilidades de pensamiento creativo en las formas de abordaje. Proponemos incluir la reflexividad como proceso metodológico nuclear dentro de cualquier competencia, dado que está implicada a la hora de saber utilizar las diferentes formas de pensar y conjugarlas tanto en la comprensión y estudio de la realidad social, como en la toma de decisiones y diseño de intervenciones sociales.

En este sentido, ponemos de relieve el déficit existente en el desarrollo competencial desde el marco universitario. Este instrumento no mide la capacidad de análisis global de un sujeto vinculado al desarrollo profesional, fin último del desarrollo competencial. Si no que únicamente mide la capacidad de análisis desarrollada delante de un ejercicio teórico. El docente y el estudiante se enfrentan al reto de transferir el contenido del ejercicio al contexto real de práctica. Esta distancia evidente puede compensarse por la coexistencia de otras variables como la vinculación y conocimiento del docente del ámbito del trabajo social, las claves madurativas del estudiante, relacionadas con su experiencia laboral, estudios previos realizados, otras capacidades cognitivas, etc. En este sentido, la validación del instrumento en los contextos de práctica profesional queda abierta como una nueva línea de continuidad en nuevos proyectos.

\section{Referencias bibliográficas}

Arendt, H. (1993). La condición humana. México: Paidós.

Bárcena, F. (2005). La experiencia reflexiva en educación. Barcelona: Paidós.

Bay,U. i Macfarlane, S. (2011). Teaching Critical Reflection: A Tool for Transformative Learning in

Social Work? Social Work Education, 30 (7), 745-758.

Biemans, H., Nieuwenhuis, L., Poell, R., Mulder, M. y Wesselink, R. (2004). Competence-based vet 
in the Netherlands. Journal of Vocational Education and Training, 56 (4), 523-538. doi: $10.1080 / 13636820400200268$.

Brookfield, S. (2009). The concep of critical reflection: pormises and contradictions. European Journal of Social Work, 12 (3). 293-304. doi: 10.1080/13691450902945215.

Consejo General del Trabajo Social. (2013). Informe ISSE de la investigación. La opinión de los trabajadores sociales sobre su labor profesional y los Servicios Sociales en España. Madrid: Consejo General del Trabajo Social.

D’Cruz, H., Gillingham, P. y Meléndez, S. (2007). Reflexivity, its meanings and relevance for Social Work: a critical review of the literature. British Journal of Social Work. 37 (1), 73-90. doi:10.1093/bjsw/bcl001.

Dewey, J. (1998). Cómo pensamos. Nueva exposición de la relación entre el pensamiento reflexivo y proceso educativo. Barcelona: Paidós.

Gairín, J. (ed). (2009). Guia per a l'avaluació de competències en l'àrea de ciències socials.

Agència per a la Qualitat del Sistema Universitari de Catalunya. Barcelona. Disponible en: www.aqu.cat.

García San Pedro, M. J. y Gairín, J. (2011). Los mapas de competencias: una herramienta para mejorar la calidad de la formación universitaria. Revista Iberoamericana sobre Calidad, Eficacia y Cambio en Educación. 9 (1), 84-102. Disponible en: http://www.rina ce.net/reice/numeros/arts/vol9num1/art5.pdf.

Gardner, H. (2005). Las cinco mentes del futuro: un ensayo educativo. Barcelona: Paidós.

Ginesta, M. (2011). Reflexions sobre el treball social als serveis socials bàsics. Revista de Treball Social i Serveis Socials, 194, 40-45.

González, J. y Wagenaar, R. (2003). Tuning Educational structures in Europe. Bilbao: Universidad de Deusto.

Ibarz, J., Prat, N. y Vives, T. (2013). Estudi previ de la CPA a partir d'un exercici realitzat per l'alumnat de Treball Social. Una primera aplicació de la rúbrica efectuada sobre un cas real. Recuperado de: http://hdl.handle.net/2445/49286.

Hager, P., Holland, S. y Becket, D. (2002). Enhancing the learning and employability of graduates: the role of generic skills. Business/Higher Education Round Table. Disponible en: http://www.bhert.com/publications/position-papers/B-HERTPositionPaper09.pdf (Consultado el 23 de febrero de 2014).

Hawes B., Gustavo (2004). Evaluación: estàndares y rúbricas. (Proyecto Mecesup TAL101). Recuperado a Universidad de Talca, Unidad de Gestión, Seguimiento y Evaluación Disponible en: http://www2.sep.ucr.ac.cr/GESTION/TALCACHILE.html.

Jordan, B. (2011). Austeridad y transformaciones en la política social y el trabajo Social. Documentación Social, 163, 213-232.

Llobet, M. y Vilà, G. (2013). El pensament analitic a l'ensenyament de Treball Social. Proposta de definició i conceptualització. Recuperado de: http://hdl.handle.net/2445/46526.

Moya, J. (ed.). (2008). Proyecto Atlántida. De las competencias básicas al currículo integrado. Madrid: Proyecto Atlántida.

Moya, J. (2013) El treball social davant els efectes de la crisi económica. Cap a un nou model d'intervenció. Revista de Treball Social, 200, 36-45.

Pastor, E. y Martínez, M. A. (2014). El trabajo social ante el reto de la crisis y la Educación Superior A. En: E. Pastor y M. A. Martínez. (ed.), Trabajo Social en el siglo XXI. Una perspectiva internacional comparada, (pp. 13-21). Madrid: Grupo 5.

Picornell- Lucas, M.A. (2014). La coevaluación de competencias en el Grado de Trabajo Social mediante el uso de la rúbrica. AZARBE. Revista Internacional de Trabajo Social y Bienestar Social, 3, 7-14.

Perrenoud, P. (2004). Desarrollar la practica reflexiva en el oficio de enseñar. Barcelona. Crítica y Fundamentos. Graó.

Rekalde, I. (2011). ¿Cómo afrontar el trabajo fin de grado? Un problema o una oportunidad para culminar con el desarrollo de las competencias Revista Complutense de Educación. 22 (2), 179193 http://dx.doi.org/10.5209/rev_RCED.2011.v22.n2.38488. 
Trevelyan, C., Crath, R. y Chambon, A. (2014). Promoting Critical Reflexivity through Arts-Based Media: A Case Study. British Journal of Social Work, 44 (1), 7-26 doi: 10.1093/bjsw/bcs090

Villa, A. y Poblete, M. (2007). Aprendizaje basado en competencias. Una propuesta para la evaluación de las competencias genéricas. Bilbao: Universidad de Deusto.

Yepes,M., LLobet, M. y Prat, N. (2012). Fils conductors: Transversalitat docent i pensament analític a Treball Social. Congrés Internacional de Docència Universitària i Innovació (CIDUI). Barcelona: ISBN: 978-84-695-4073-2.

Zabalza, M. G. (2003-2004). Innovación en la enseñanza Universitaria. Contextos Educativos, 6-7. 113-136.

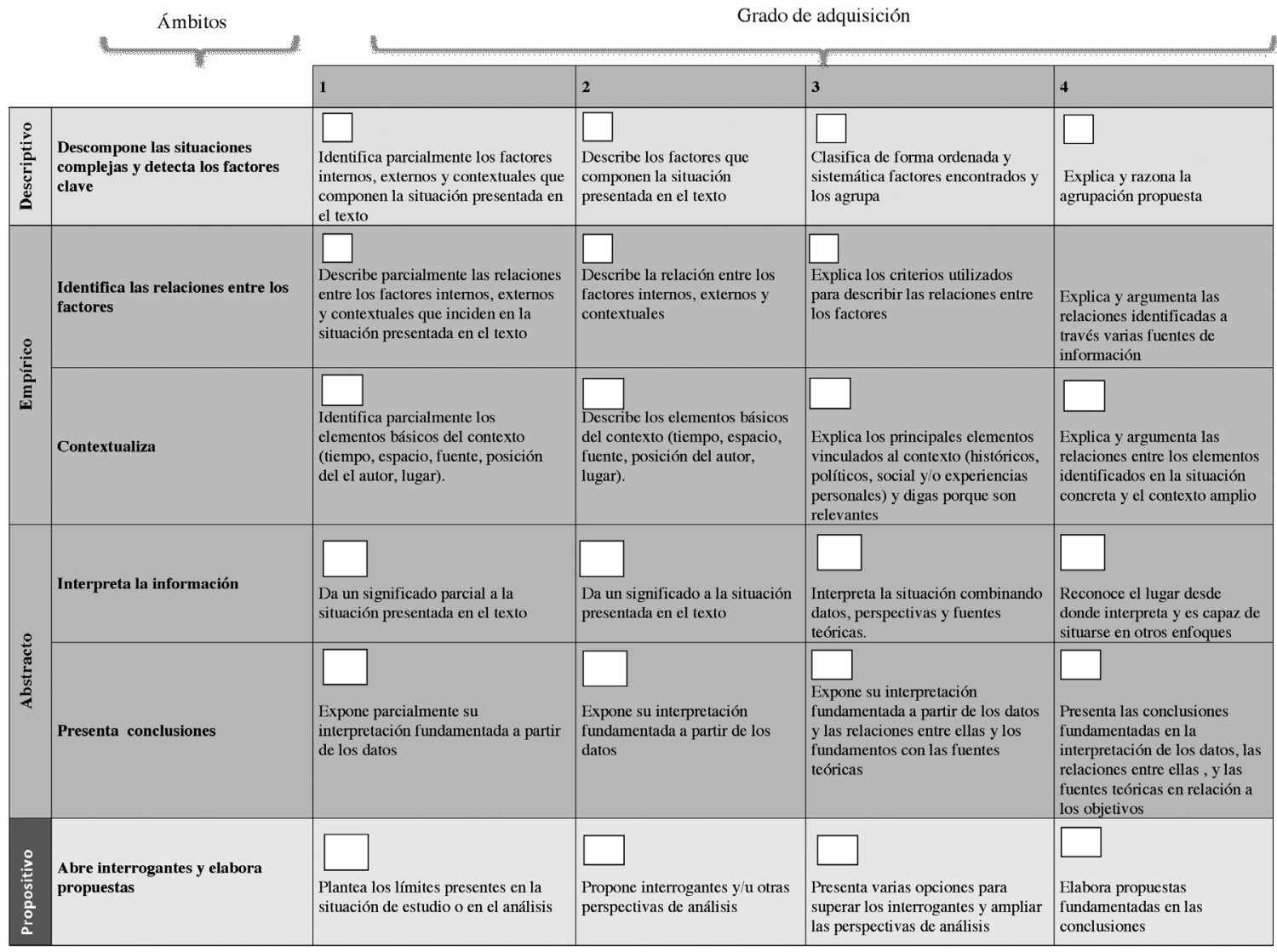

Anexo I. Rúbrica de aprendizaje y de evaluación de la competencia de pensamiento analítico en el Grado de Trabajo Social. 